\title{
Relationships of A1c Variability for 10 Years and Beta Cell Function at the Time of Diagnosis in Type 2 Diabetes
}

Eun Ju Lee and Mi-Kyung Kim*

Department of Internal Medicine, College of Medicine, Inje University, Republic of Korea

*Corresponding author: Mi-kyung Kim, M.D., Ph.D., Associate professor, Chair, Endocrinology Diabetes and Metabolism, Internal Medicine, Haeundae Paik Hospital, College of Medicine, Inje University, 875, Hauendae-ro, Hauendae-gu, Busan, 612-862,Republic of Korea, Tel: 82517970636 ; Fax: 8251 797 2070; E-mail: kmkdoc@hanmail.net

Rec date: March 16, 2015 Acc date: April 13, 2015 Pub date: April 20, 2015

Copyright: (C) 2015 Lee EJ, et al. This is an open-access article distributed under the terms of the Creative Commons Attribution License, which permits unrestricted use, distribution, and reproduction in any medium, provided the original author and source are credited.

\begin{abstract}
Background: Recent several reports emphasized A1c variability along with average blood glucose determined by Alc. Many studies have been showing the relationship of A1c variability with chronic diabetic complications. But, associations between patterns or degrees of A1c variability and influential factors have not been studied much. Therefore, this study aimed to evaluate the relationships of A1c variability for 10 years and beta cell function at the time of diagnosis in type 2 diabetes in clinical practice.
\end{abstract}

Methods: Subjects of this study were 518 newly diagnosed type 2 diabetic patients. To evaluate beta cell function, we checked fasting c-peptide, glucose, and insulin and assessed homeostasis model assessment beta cell function index (HOMA- $\beta$ ) and homeostasis model assessment for insulin resistance (HOMA-IR) at the time of diagnosis. A1cs, fasting plasma glucose, and lipid profile were serially measured at least once in every year for 10 years.

Results: We found that higher A1c variability was associated with younger age at diagnosis of diabetes, lower BMI, higher first A1c, higher 2nd year A1c, and higher mean A1c. C-peptide and homeostasis model assessment beta cell function index were the highest in the low SD group and the lowest in high SD group.

Conclusion: A1c variability for 10 years in newly diagnosed type 2 diabetes is inversely related with the marker of beta cell function at the time of diagnosis.

Keywords: A1c Variability; Beta cell function; Type 2 diabetes

\section{Introduction}

Alc is an important parameter of average blood glucose over several months in diabetic patients. It is well known that a level of glycemic control determined by Alc is an important marker for chronic complications of diabetes. Many studies have reported that lowering Alc reduces the risk and progression of diabetic complications $[1,2]$.

In recent years, along with average blood glucose determined by Alc, glycemic variability has also been emphasized. The authors of DCCT study first suggested the role of glycemic variability in diabetic complications [3]. Since then, many in vivo and in vitro studies showed glucose fluctuation induced higher oxidative stress than sustained hyperglycemia, leading to dysfunction of vascular endothelium, renal mesangial and tubulointerstitial cell [4]. Our group also previously reported the effect of glucose fluctuation on pancreatic beta cell apoptosis and its mechanism [5,6]. These studies partially explained the relationship of glucose variability with chronic diabetic complications.

Recently, studies on A1c variability reflecting long-term glucose variability as well as acute glucose variability assessed with continuous glucose monitoring system (CGMS) have been published [7-9]. The long term variability (expressed as A1c variability) may be more important than short-term glucose variability as a risk marker for diabetic complications because atherosclerosis is not a short-term event. Although there is a lack of evidence on the relationship between Alc variability and diabetic macrovascular complications, many studies have showed the relationship of glucose variability with chronic diabetic complications [7-11] and a recent retrospective cohort study reported that $\mathrm{A} 1 \mathrm{c}$ variability can predict all-cause mortality in type 2 diabetic patients, independent of mean A1c and other confounders [9].

Glycemic variability in type 2 diabetes results from the complex interplay between pathophysiological factors, behavioural and treatment factors. Glycemic variability can be influenced by variable factors such as diet, exercise, medication and compliance during treatment. Unstable glucose fluctuations are more frequently shown in patients with type 1 diabetes than patients with type 2 diabetes and in patients with advanced type 2 diabetes in clinical practice. Therefore, the degrees of endogenous beta cell dysfunction in type 2 diabetes at the time of diagnosis may be one of important predictors for future glucose and Alc variability. There were few studies on the association of glucose variability with pancreatic beta cell dysfunction, but most of the studies had assessed the glucose variability with continuous glucose monitoring reflecting short-term glucose variability and evaluated the association with postprandial beta cell dysfunction [12]. In type 2 diabetes, the association between the degrees of glycemic variability and pancreatic beta cell dysfunction at the time of diagnosis has not been studied yet. Therefore, we investigated the Alc variability during the first 10 years after diagnosis of type 2 diabetes and evaluated the 
Page 2 of 4

relationship between glycemic variability and beta cell function at the time of diagnosis in type 2 diabetes.

\section{Methods}

Inclusion criteria of our study were type 2 diabetes followed up in our institute for at least 10 years. All type 2 diabetic subjects were eligible to be recruited unless they were type 1 diabetic patients. Clinical data of 518 newly diagnosed diabetic patients including their baseline characteristics, including age, sex, height and weight were retrospectively collected. Body mass index (BMI) was calculated as

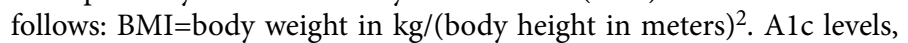
fasting plasma glucose, total cholesterol, triglycerides, low-density lipoprotein (LDL) cholesterol, and high-density lipoprotein (HDL) cholesterol were measured serially at least once in every year for 10 years since the patients were diagnosed as diabetes. At the time of diagnosis with type 2 diabetes, fasting c-peptide and insulin levels were assessed. For biochemical measurements, sampling was performed in the morning after an overnight fast (for $\geq 8 \mathrm{~h}$ ). Serum fasting c-peptide and insulin were measured using a radioimmunoassay. The fasting glucose levels were measured with hexokinase method and hemolysed blood samples were excluded.

Intrapersonal SD of serially measured $\mathrm{A} 1 \mathrm{c}$ was adjusted for a different number of assessments among patients using the equation of (adj-Alc-SD $=S D / \sqrt{ }[n /(n-1)])$ and was used to express variability of A1c.
To evaluate beta cell function, we checked fasting c-peptide, glucose, and insulin and assessed homeostasis model assessment beta cell function index (HOMA- $\beta$ ) and homeostasis model assessment for insulin resistance (HOMA-IR) at the time of diagnosis.

Continuous variables are expressed as means \pm SD if normally distributed. Categorical variables are expressed as percentages. Differences between patient groups were analyzed by Student $t$ test (two groups) or ANOVA (over two groups) for normally distributed continuous variables. The chi-squared $\left(\chi^{2}\right)$ test was used for categorical variables. SPSS version 18.0 was used for statistical calculations. $p$ $<0.05$ was considered statistically significant. Institutional Review Board was obtained by our institute.

Results

\section{Clinical and biochemical characteristics}

Of the 518 patients, $46.5 \%$ of patients were men and the mean age was $53.0 \pm 12.2$ years. To assess which patient characteristics were associated with Alc variability during the follow-up, we divided the patients into tertiles of intrapersonal SD of serially measured Alcs. Patients in the lowest, the middle and the highest tertile were referred as the low SD group, the middle SD group and the high SD group, respectively. The characteristics of these three groups were summarized in Table 1.

\begin{tabular}{|l|l|l|l|}
\hline \multirow{2}{*}{} & \multicolumn{2}{l}{ SD of serial A1c } & High \\
\cline { 2 - 4 } & Low & Middle & 176 \\
\hline No. & 171 & 171 & $52.9 \pm 12.9^{\mathrm{a}}$ \\
\hline BMI (kg/m²) & $55.6 \pm 11.5$ & $52.9 \pm 11.7^{\mathrm{a}}$ & $23.8 \pm 3.2^{\mathrm{a}}$ \\
\hline Mean SD of A1c & $25.0 \pm 2.89$ & $24.2 \pm 3.4$ & $1.79 \pm 1.26^{\mathrm{a}, \mathrm{b}}$ \\
\hline Mean First A1c (\%) & $0.54 \pm 0.15$ & $0.95 \pm 1.12^{\mathrm{a}}$ & $9.01 \pm 2.64^{\mathrm{a}, \mathrm{b}}$ \\
\hline Mean 2nd year A1c (\%) & $6.47 \pm 1.17$ & $7.36 \pm 1.45^{\mathrm{a}}$ & $7.02 \pm 1.84^{\mathrm{a}, \mathrm{b}}$ \\
\hline Mean (10yr) A1c (\%) & $6.21 \pm 1.06$ & $6.66 \pm 1.28^{\mathrm{a}}$ & $7.78 \pm 1.07^{\mathrm{a}, \mathrm{b}}$ \\
\hline Total cholesterol (mg/L) & $6.48 \pm 0.91$ & $7.14 \pm 0.78^{\mathrm{a}}$ & $194.8 \pm 55.8$ \\
\hline LDL cholesterol (mg/L) & $190.5 \pm 30.9$ & $198.0 \pm 42.9$ & $126.8 \pm 47.4$ \\
\hline HDL cholesterol (mg/L) & $120.3 \pm 29.2$ & $125.8 \pm 39.7$ & $46.8 \pm 15.1$ \\
\hline Triglyceride (mg/L) & $47.6 \pm 13.5$ & $48.2 \pm 13.2$ & $172.3 \pm 124.7$ \\
\hline
\end{tabular}

Table 1: The characteristics of patients divided into tertiles of intrapersonal SD of serially measured A1cs for $10 y e a r s .{ }^{a} \mathrm{p}<0.05 \mathrm{vs}$ low SD group, ${ }^{\mathrm{b}} \mathrm{p}$ $<0.05$ vs middle SD group

The patients in the high SD group were younger (55.6 \pm 11.5 vs 52.9 \pm 12.9 years) and had lower BMI than those in the low SD group ( 25.0 \pm 2.89 vs $23.8 \pm 3.2 \mathrm{~kg} / \mathrm{m}^{2}$ ). The mean A1c levels of the low, middle and high SD group at the time of the diagnosis were $6.47 \pm 1.17 \%, 7.36$ \pm 1.45 and $9.01 \pm 2.64$, respectively. The mean A1c levels of three groups at the second year were also significantly different $(6.21 \pm 1.06$, $6.66 \pm 1.28,7.02 \pm 1.84, \mathrm{p}<0.05)$. The mean A1c level for 10 years was the highest in the high A1c SD group and the lowest in the low A1c SD group. Total cholesterol, LDL cholesterol, HDL cholesterol and triglyceride levels did not differ among the groups.

\section{Mean Alc and Alc variability}

The patients were divided into 3 groups according to the range of the mean Alc value to evaluate the association between the mean Alc and Alc variability (Figure 1 ). In the patient group with the mean Alc for 10 years less than $7 \%, 51.7 \%$ of low SD of serial A1c were included. 
In the group with the mean A1c over 9\%, 78.9\% of high SD group were included. There were significant differences among three groups $(\mathrm{p}<0.001)$.

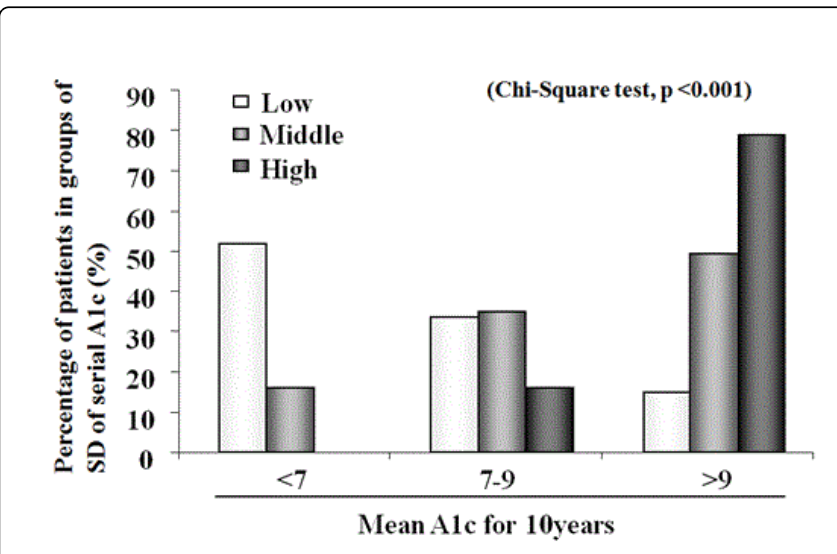

Figure 1: The tertiles of SD of serial Alc among the groups divided according to mean A1c values $(<7,7-9,>9)$.

\section{Relationships between A1c variability and beta cell function}

We compared fasting baseline c-peptide, HOMA- $\beta$ and HOMA-IR among the SD groups to evaluate the relationship between A1c variability and beta cell function at the time of diagnosis. Fasting baseline c-peptide and HOMA- $\beta$ at the time of diagnosis were significantly different among the three groups $(\mathrm{p}<0.05)$, but HOMA IR did not significantly differ. C-peptide and HOMA- $\beta$ were the highest in the low SD group and the lowest in high SD group (Figure 2a-2c).

\section{Discussion}

This study was conducted to investigate the relationship of A1c variability with beta cell function in type 2 diabetes in real clinical practice. Recently, glycemic variability has also been emphasized and Monnier et al. have emphasized that glycemic vavriability is more important than chronic sustained hyperglycemia contributing to the development of secondary diabetes complications [13]. A1c variability relates to blood glucose changes over long periods of time. Increasing attention has been focused on A1c variability reflecting long-term glucose variability and diabetic complications and a number of studies have revealed the evidence on the relationship between A1c variability and diabetic complications in type $1 \mathrm{DM}$ and type $2 \mathrm{DM}$. A retrospective analysis of the DCCT dataset had showed that variability in $\mathrm{AlC}$ adds to the mean value in predicting microvascular complications in type 1 diabetes. In that study, Alc variability is an independent risk factor for nephropathy and retinopathy independently of mean $\mathrm{HbAlc}$ in type 1 diabetic patients [7]. And, a report from the Finnish Nephropathy Study Group showed that A1c variability was not only predictive of incident microalbuminuria and progression of renal disease but also of incident CV disease events [8]. Also, $\mathrm{HbA1c}$ variability was shown to be an independent variable that increased the effect of HbAlc on the risk of microalbuminuria in adolescent patients with T1DM in s the Oxford Regional Prospective Study and the Nephropathy Family Study [14]. There were several studies on the associations between the Alc variability and diabetic complications in type $2 \mathrm{DM}$. Alc variability affected the development of microalbuminuria independently of mean Alc in type 2 diabetes in the Tsukuba Kawai Diabetes Registry study [11]. A1c variability, even measured as early as 2 years, was independently associated with the development of microalbuminuria in a Taiwanese study [15]. Also, very recently in the Renal Insufficiency And Cardiovascular Events (RIACE) Italian Multicenter Study, Alc standard deviation was associated with macroalbuminuria and albuminuric stages 3-5 of chronic kidney disease, independently of average HbAlc, even after adjustment for other known predictors of diabetic nephropathy, whereas average $\mathrm{HbA} 1 \mathrm{c}$ was not $[16,17]$. a)

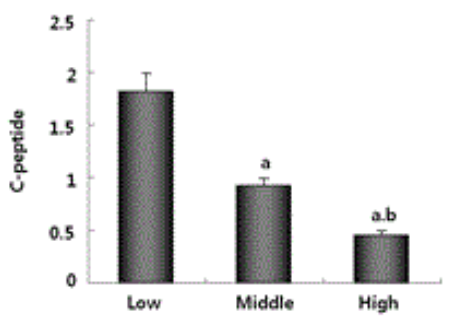

b)

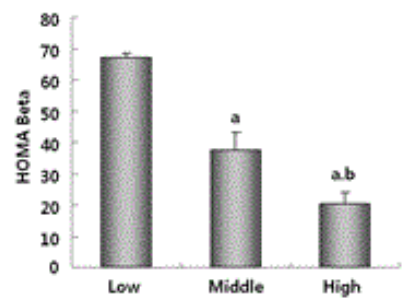

c)

Figure 2 a-2c: The comparison of fasting baseline HOMA-IR, HOMA- $\beta$ and c-peptide among groups

We tried to find the factors affecting the Alc variability which have been shown the association with diabetic complications and the higher Alc variability was associated with younger age at diagnosis of diabetes, lower BMI, higher first A1c, higher 2nd year A1c, and higher mean Alc in our result. Associations between patterns or degrees of Alc variability and influential factors have not been studied much. Several studies suggested that multiple factors including age, diabetic duration, insulin sensitivity, and other metabolic profiles might be associated with $\mathrm{A} 1 \mathrm{C}$ variability. In type 1 diabetes, higher variability was associated with younger age, lower age at onset of diabetes, shorter duration of diabetes, lower insulin sensitivity, dyslipidemia, higher baseline A1c, both current and past smoking history, and lower socioeconomic status [8]. In another study with type 2 diabetic patients, subjects with lower Alc variability had more favorable 
metabolic profiles, including BMI, waist circumference, systolic and diastolic blood pressure, total cholesterol, triglyceride, LDL-cholesterol and ALT, than those with higher A1c variability [9]. In our results, higher A1c variability was associated with younger age, lower BMI and higher mean A1c. The reason that younger patients have higher A1c variability may be because of younger patients' suboptimal management of diabetes (including of irregular life style). According to a study which analyzed changes in A1c during the first 6 years after diagnosis of type 2 diabetes, age was the only long-term predictor for changes in A1c [18]. A sharp rise in a long-term glycemic level was observed in a quarter of the patients, especially in the relatively young. In a previous study, it was found that lower BMI was associated with lower A1C variability and it was explained by mild insulin resistance. Nevertheless, lower BMI was associated with higher A1C variability in our study. This result can be explained by our other result that the patients with less endogenous beta cell function at the time of diagnosis showed the larger glucose variability.

And, we can find that Alc variability was inversely related with the marker of beta cell function at the time of diagnosis in our result. There were few studies on the association of glucose variability with pancreatic beta cell dysfunction. One study reported a nonlinear relationship between glycemic variability and beta cell dysfunction in type 2 diabetic patients [12]. In that study, short-term glucose variability assessed with continuous glucose monitoring system (CGMS) was associated with postprandial beta cell function. Another reported that glucose variability was significantly reduced in youth with short-term type 1 diabetes who retained residual $\beta$-cell function than in youth with type 1 diabetes for a longer duration [19]. However, we could not find the studies showing relationships between A1C variability and $\beta$-cell function at the time of diagnosis in type 2 diabetes. Our results supported that long-term glucose variability might have a correlation with endogenous beta cell defect at the time of diagnosis but not with insulin resistance. Based on the relationships between A1c variability and beta cell defect, we may also explain why young age and lower BMI are correlated with higher A1c variability.

There are some limitations in our study. This study was retrospectively conducted in a single clinic in Korea. And, there was no comprehensive analysis for hyperglycemic treatment modalities. We did not have information about the medications and the change of them for 10 years since our study is retrospective. We believe that physicians did their best to achieve optimal glycemic control in real practice. In other words, our results may represent real world status, not study setting.

In summary, Alc variability for 10 years in newly diagnosed type 2 diabetes is inversely related with the marker of beta cell function at the time of diagnosis. The Alc variability in type 2 diabetes may be reflected by dysfunction of the pancreatic beta cell at the time of diagnosis. We think that it is important to be aware of the association of these and applied to personalized treatment in type 2 diabetes.

\section{References}

1. Diabetes Control and Complications Trial Research Group (1993) The effect of intensive treatment of diabetes on the development and progression of long-term complications in insulin-dependent diabetes mellitus. The Diabetes Control and Complications Trial Research Group. N Engl J Med 329: 977-986.

2. UK Prospective Diabetes Study (UKPDS) Group (1998) Intensive blood glucose control with sulphonylureas or insulin compared with conventional treatment and risk of complications in patients patients with type 2 diabetes. Lancet 1352: 837-853.

3. The diabetes Control and Complication Trial Research Group (1995) The relationship of glycemic exposure (HbAlc) to the risk of development and progression of retinopathy in the diabetes control and complications trial. Diabetes 44: 968-983.

4. Ceriello MA, Ihnat (2010) Glycaemic variability: a new therapeutic challenge in diabetes and the critical care setting. Diabet. Med 27: 862867.

5. Kim M, Jung H, Yoon C, Ko J, Jun H, et al. (2010) The Effect of Glucose Fluctuation on Apoptosis and Function of INS-1 Pancreatic Beta Cells. Korean Diabetes J 34: 47-54.

6. Kim M, Chung H, Yoon C, Lee E, Kim T, et al. (2012) Increase of INS-1 cell apoptosis under glucose fluctuation and the involvement of FOXOSIRT pathway. Diabetes Res Clin Pract 98: 32-139.

7. Kilpatrick ES, Rigby AS, Atkin SL (2008) A1C variability and the risk of microvascular complications in type 1 diabetes: data from the Diabetes Control and Complications Trial. Diabetes Care 31: 2198-2202.

8. Waden J, Forsblom C, Thorn LM, Gordin D, Saraheimo M, et al. (2009) A1C Variability Predicts Incident Cardiovascular Events, Microalbuminuria, and Overt Diabetic Nephropathy in Patients With Type 1 Diabetes. Diabetes 58: 2649-2655.

9. Ma WY, Li HY, Pei D, Hsia TL, Lu kc, et al. (2012) Variability in hemoglobin Alc predicts all-cause mortality in patients with type 2 diabetes. Journal of Diabetes and Its Complications 26: 296-300.

10. Kilpatrick ES, Riqby AS, Atkin SL (2009) Effect of Glucose Variability on the Long-Term Risk of Microvascular Complications in Type 1 Diabetes. Diabetes Care 32: 1901-1903.

11. Sugawara A, Kawai K, Motohashi S, Saito K, Kodama S, et al. (2012) HbA1c variability and the development of microalbuminuria in type 2 diabetes: Tsukuba Kawai Diabetes Registry 2. Diabetologia 55: 2128-2131.

12. Kohnert KD, Augstein P, Zander E, Heinke P, Peterson K, et al. (2009) Glycemic Variability Correlates Strongly With Postprandial beta-Cell Dysfunction in a Segment of Type 2 Diabetic Patients Using Oral Hypoglycemic Agents. Diabetes Care 32: 1058-1062.

13. Monnier L, Mas E, Ginet C, Michel F, Villon L, et al. (2006) Activation of oxidative stress by acute glucose fluctuations compared with sustained chronic hyperglycemia in patients with type 2 diabetes. JAMA 295: 16811687

14. Marcovecchio ML, Dalton RN, Chiarelli F, Dunger DB (2011) A1C variability as an independent risk factor for microalbuminuria in young people with type 1 diabetes. Diabetes Care 34: 1011-1013

15. Hsu CC, Chang HY, Huang MC, Hwang SJ, Yang YC, et al. (2012) HbAlc variability is associated with microalbuminuria development in type 2 diabetes: a 7-year prospective cohort study. Diabetologia 55: 3163-3172.

16. Cavalot F (2013) Do data in the literature indicate that glycaemic variability is a clinical problem? Glycaemic variability and vascular complications of diabetes Diabetes, Obesity and Metabolism Suppl 2: 3-8

17. Penno G, Solini A, Bonora E, Fondelli C, Orsi E et al. (2013) HbA1c variability as an independent correlate of nephropathy, but not retinopathy, in patients with type 2 diabetes: the Renal Insufficiency And Cardiovascular Events (RIACE) Italian Multicenter Study. Diabetes Care 36: $2301-2310$

18. Olivarius Nde F, Siersma V, Hansen LJ, Drivsholm T, Horder (2009) Changes in levels of haemoglobin Alc during the first 6 years after diagnosis of clinical type 2 diabetes. Scandinavian Journal of Clinical \& Laboratory Investigation 69: 851-857.

19. Sherr J, Tamborlane WV, Xing D, Tsalikian E, Mauras N, et al. (2012) Achievement of Target A1C Levels With Negligible Hypoglycemia and Low Glucose Variability in Youth With Short-Term Type 1 Diabetes and Residual $\beta$-Cell Function. Diabetes Care 35: 817-820. 